\title{
Incidence of Venous Thromboembolism Following Below Knee Plaster Immobilization
}

\author{
A.E.Abdelmonem, M.G.Elashhab and A.A.Elsheikh \\ Orthopaedic surgery, Dept., Faculty of Medicine, Benha Univ., Benha, Egypt \\ Email: ahmed.abdelmonem@nhs.net
}

\begin{abstract}
Background: Venous thromboembolism (VTE) has an incidence between 0.7 and 2.69 per 1000 per year. Temporary lower limb immobilisation after an injury is a significant contributor to Deep venous thrombosis (DVT). Previous studies showed an incidence rate between $1 \%$ to $20 \%$. However, the real incidence of DVT in patients with isolated stable ankle, foot fractures and sprains treated conservatively is not well known. Objectives: This study aims to assess the incidence of VTE after lower limb plaster immobilisation and if we should consider giving chemoprophylaxis. Patients and methods: A prospective cross-sectional study included consecutive twenty patients with stable ankle, foot fractures, and sprains managed conservatively with below-knee plasters. There was no national/local policy to offer VTE prophylaxis. VTE Risk assessments were done and documented. After Plaster removal, all patients had a Doppler ultrasound examination (DUE) to confirm or rule out VTE diagnosis. Results: Twenty patients (11men/ 12 women) were followed up for at least six weeks. The Mean age was 46.30 17.50 (Range 18-70) years old. Twelve patients had below-knee cast $(60 \%)$, Eight had below knee slab (40\%). The mean immobilisation duration was $38.50 \pm 15.8$ (Range 15-62). Thirteen patients (65\%) had an increased risk for VTE. One patient (5\%), 28 years female, had DVT, scoring only one point of VTE risks (oral contraceptive pills). She was a non-smoker with, BMI of 26. She was diagnosed with a popliteal DVT after removing a below-knee slab for ankle sprain at two weeks post-injury and treated accordingly with no complication. Conclusion: Incidence of DVT after below-knee cast immobilisation about $5 \%$. Similar patients should be assessed, and proper prophylaxis is given to decrease the morbidity and mortality of having VTE
\end{abstract}

Keywords: Venous Thromboembolism, below Knee Plaster Immobilization, Doppler ultrasound examination.

\section{Introduction}

Venous thromboembolism (VTE) has a background incidence of between 0.7 and 2.69 per 1000 per year [1]. Deep vein thrombosis (DVT) approximately three times more often than pulmonary embolism.

Venous thromboembolism is a serious condition that may lead to chronic morbidities, such as postthrombotic syndrome, pulmonary hypertension, and increased mortality. Post-thrombotic syndrome is seen in half of the patients with symptomatic DVT [8]. Around $4 \%$ of patients with PE develop chronic pulmonary hypertension within two years [9]. The mortality rate of venous thrombosis is estimated to be $1.8 \%$ in the first month in non-cancer patients after DVT and $6.8 \%$ after PE.

Risk factors are either permanent or transient. Permanent risk factors include thrombophilia, cancer, increasing age, family or personal history of deep vein thrombosis and increasing body mass index. Transient risk factors include major surgery, postpartum state, oral contraceptive pill, hormone replacement therapy, pregnancy, trauma and immobility, smoking, dehydration and one or more significant comorbidity (e.g. heart disease, metabolic or endocrine diseases). [2]

In clinical practice, the Wells Criteria [3] is often utilised to stratify the risk of a patient clinically suspected of having a DVT; it is not a diagnostic test. The scoring system guides what to do next for the patient investigations (D-dimer or US Doppler imaging). However, this scoring system serves as evidence-based medicine and is conducted based on the study of risk factors for DVT. Despite not being all-inclusive, it provides a broad grouping of the most common risk factors.

The criteria are as follows: active cancer, or cancer treatment, bedridden for more than three days, major surgery within the last four weeks, calf swelling greater than $3 \mathrm{~cm}$ more than contra-lateral leg $10 \mathrm{~cm}$ below tibial tuberosity, collateral superficial veins present (varicose veins), diffuse leg swelling, localized tenderness along with the deep venous system, pitting oedema, paralysis, paresis, immobilisation of lower extremity, previous DVT, and lack of other more likely aetiology.

Most stable fractures of the ankle and foot are treated conservatively in a below-knee plaster. It has been believed that this method of immobilisation may increase the risk of deep-vein thrombosis (DVT) because of the inactivation of the calf pumps [4]. DVT usually goes discreet and dissolves on its own. But some cause symptoms such as redness of the skin, calf swelling and pain. DVT can become dangerous if the blood clot dislodges from the vein and travels through the veins into the lungs, causing pulmonary embolism.

The risk for venous thromboembolism (VTE) associated with lower limb immobilisation is unclear, owing to a lack of evidence from studies in this patient group. However, previous studies have indicated that DVT incidence ranges between $1.1 \%$ and $20 \%$ in various lower limb injuries treated in a plaster cast. Moreover, asymptomatic DVT affects between 4 and $40 \%$ of patients; symptomatic DVT affects 1 in 250 , symptomatic pulmonary embolism affects 1 in 500, with fatal pulmonary embolism affecting 1 in 15,000 
within 90 days of lower limb cast immobilisation.[4,5]

Routine thromboprophylaxis for these patients is not indicated in international VTE guidelines yet is considered standard in some centers [6].

NICE guidelines recommend VTE prophylaxis to patients with below-knee casts following foot and ankle surgery following risk assessment [2]; there are no guidelines about VTE prophylaxis to patients in below-knee plasters without surgeries. [7]

This study aimed to assess Venous Thromboembolism (VTE) incidence after lower limb plaster immobilisation and consider giving chemoprophylaxis.

\section{Patient and Method}

A prospective cross-sectional study was done, including twenty patients with stable ankle, foot fractures, and sprains managed conservatively with below-knee plasters. The study was approved by the institutional Ethics Committee, and written informed consent was obtained from each patient.

Inclusion criteria: patients with isolated foot or ankle fractures and sprains treated conservatively (below knee slab or plaster cast), 18 years age or over, both males and females and consent was obtained from all participated patients

Exclusion criteria: Patients with associated lower-limb fractures require surgery and patients with comorbidity that require prophylactic LMWH or oral anticoagulants.

Risks assessment were noted down, which include (Age, dehydration, obesity, history of DVT, varicose veins, pregnancy, smoking, hormone replacement therapies, known thrombophilia and active cancer or cancer treatment.

Patient age, sex, occupation, residency, religion, education, marital status, health and disability status, psychiatric diagnosis as well as contact information was collected and noted down separately
During plaster immobilisation, clinical orthopaedic and radiological reviews were done, and clinical examination and observation for any symptoms or signs suggestive of DVT were noted.

After Plaster removal, all patients were referred for Doppler ultrasound examination (DUE) to confirm or rule out the diagnosis of silent DVT. Finally, the incidence of DVT was calculated, and the roles of predisposing factors were investigated.

\section{Results}

The study included twenty patients. All patients were followed up for at least six weeks.

The Mean age was 46.30 \pm 17.50 (Range 18-70) years old. $55 \%$ were males.

Twelve patients had a below-knee cast $(60 \%)$, while Eight had below-knee back-slab (40\%).

The mean immobilisation duration was 38.50 \pm 15.8 (Range 15-62), with eight patients allowed to bear weight fully, 8 to bear weight partially, and the remaining four were kept non-weight-bearing. Of those twenty patients, thirteen had a risk For VTE $(65 \%)$. The incidence of VTE was $5 \%$ (1 of 20 Patients)

The mean body mass index (BMI) of the patients was $28 \mathrm{~kg} / \mathrm{m} 2$ (17.9 to 51 ) with $41 \mathrm{~kg} / \mathrm{m} 2$ being overweight (BMI > $25 \mathrm{~kg} / \mathrm{m} 2)$ and $24 \mathrm{~kg} / \mathrm{m} 2$ obese $(\mathrm{BMI}>30 \mathrm{~kg} / \mathrm{m} 2)$.

Two of the included patients were on Contraceptive pills and continue to take them during immobilisation duration.

A total of 5 patients were smokers and continued to smoke during the course of the treatment.

The plaster cast was removed, and the union of the fracture was assessed clinically and radiologically.

One patient (5\%) Patient with DVT was rescanned after one week to assess the progression of the thrombus.

Table (1) Nature of injuries of studied patients No. $=20$

\begin{tabular}{|c|c|c|}
\hline & Studied patients & $\mathrm{No}=\mathbf{2 0}$ \\
\hline BMI & $28.61 \pm 4.80$ & \\
\hline Mean \pm SD & $24-41.5$ & \\
\hline Rang & 27 & \\
\hline \multicolumn{3}{|l|}{ Median } \\
\hline Duration in days & $38.50 \pm 15.8$ & \\
\hline Mean \pm SD & $15-62$ & \\
\hline Rang & 44 & \\
\hline \multicolumn{3}{|l|}{ Median } \\
\hline Trauma & No & $\%$ \\
\hline \multicolumn{3}{|l|}{ Fracture Lat. Malleolus } \\
\hline Fracture medial Malleolus & 2 & 10 \\
\hline Fracture $5^{\text {th }}$ Metatarsal & 1 & 5 \\
\hline Ankle spring & 8 & 40 \\
\hline Foot spring & 6 & 30 \\
\hline \multirow[t]{2}{*}{ Calcaneal fracture } & 2 & 10 \\
\hline & 1 & 5 \\
\hline \multicolumn{3}{|l|}{ Type of plaster } \\
\hline Below knee cast & 12 & 60 \\
\hline Below knee slab & 8 & 40 \\
\hline
\end{tabular}


Table (2) Medical history of studied patients No.=20

\begin{tabular}{|c|c|c|}
\hline & Studied patients & $\mathrm{No}=\mathbf{2 0}$ \\
\hline & No & $\%$ \\
\hline \multicolumn{3}{|l|}{ Smoking } \\
\hline Yes & 9 & 45 \\
\hline No & 11 & 55 \\
\hline \multicolumn{3}{|l|}{ DM } \\
\hline Yes & 5 & 25 \\
\hline No & 15 & 75 \\
\hline \multicolumn{3}{|l|}{ HTN } \\
\hline Yes & 9 & 45 \\
\hline No & 11 & 55 \\
\hline \multicolumn{3}{|l|}{$\mathrm{HCV}$} \\
\hline Yes & 1 & 5 \\
\hline No & 19 & 95 \\
\hline \multicolumn{3}{|l|}{ Coc } \\
\hline Yes & 2 & 10 \\
\hline No & 18 & 90 \\
\hline \multicolumn{3}{|l|}{ VTE risk } \\
\hline Yes & 13 & 65 \\
\hline No & 7 & 35 \\
\hline \multicolumn{3}{|l|}{ Score } \\
\hline Mean \pm SD & $1 \pm 0.91$ & \\
\hline Rang & $0-2$ & \\
\hline Median & 1 & \\
\hline
\end{tabular}

\section{Discussion}

According to the results of this study, the incidence of DVT after below-knee cast immobilisation about $5 \%$, which seems to be low in this category; however, it's very important to consider the risk, morbidity, and mortality as well as postthrombotic complication.

Our Study results agree with S. Patil study done during 2007[7], which included about 100 patients (51 males and 49 females) with ankle fractures only and didn't include other types of orthopaedics trauma treated with below-knee plasters such as foot fractures and ligamentous injuries. The mean age was 43 years (16 to 74). The patients' mean body mass index (BMI) was $28 \mathrm{~kg} / \mathrm{m} 2$ (17.9 to 51$)$. There were 15 patients on low-dose aspirin (75 mg daily) for cardiovascular or cerebrovascular problems, and one was on depoprogesterone (implant contraceptive).

The mean duration of treatment in the plaster cast was six weeks ( 3 to 7 ), with 72 patients allowed to bear weight fully and nine to bear weight partially. All patients were assessed for DVT by duplex colour Doppler ultrasonography of the affected limb.

Ultrasonography showed that five patients, three of whom were smokers, had a DVT, none had any symptoms or signs of DVT. No formal risk assessment was done; thus, a conclusion cannot be reached about the percent of high-risk patients.

The DVT involved the peroneal vein in two patients, the peroneal and the posterior tibial veins in one, the popliteal vein, and the superficial femoral vein in the last patient. In summary, the incidence of DVT was $5 \%$.
On the other hand, a study by Jorgensen in 2002 [4] carried out a randomized, controlled study on 300 patients treated by immobilisation in a plaster cast and noted that the incidence of DVT was $18 \%$. However, this study did not mention the nature of the plaster cast (above- or below-knee) and included patients with different injuries.

The largest Randomised controlled trials [9] of which we are aware included 3680 participants in 2017 as an update to previous studies during 2008[10] and 2014[11]. They included eight Randomised controlled trials (RCTs) and controlled clinical trials (ccts). Participants were divided into three groups: the first group received Low Molecular Weight Heparin subcutaneously once per day, the second group received no preventive treatment and placebo. DVT was diagnosed by compression ultrasound, venography, or both, in participants with a leg injury who were immobilised in a plaster cast or brace for at least one week; positive DVT cases ranged from $4.3 \%$ to $40 \%$ in the control groups and $0 \%$ to $37 \%$ in the Low Molecular Weight Heparin group. The risk of DVT was lower in participants who received LMWH.

Further analysis also showed a reduction in the occurrence of DVT when the use of LMWH was compared to no treatment or placebo in the following groups of participants: conservatively treated patients below-knee casts, operated patients, patients with fractures, patients with soft-tissue injuries, patients with above-knee thrombosis, and patients with belowknee thrombosis. There were no differences noted between the group receiving LMWH and control groups for change in the incidence of pulmonary 
embolism. Additionally, fewer symptoms related to venous thromboembolism in the LMWH groups than in the control groups. One case of death due to pulmonary embolism was reported in the control group. [9]

Another large known, population-based casecontrol study [12] (4,418 cases; 6,149 controls) showed a 56-fold increased risk of venous thromboembolism in patients with below-knee cast immobilisation compared to patients with no cast. Additionally, they found a further increased risk for patients with a below-knee cast who had additional genetic or acquired risk factors (e.g. Obesity, oral contraception, factor $\mathrm{V}$ Leiden mutation, and prothrombin G20210A mutation), with relative risks ranging between $17 \%$ and $23 \%$ as compared with patients without a cast and such risk factors (all over one year following cast application). Finally, they found an accumulation of several risk factors in patients who developed a thrombotic event.

Van Adrichem Conducted another large, population-based case-control study [13] of 1519 patients who underwent randomisation, of whom 1435 were included in the intention-to-treat population. Venous thromboembolism occurred in 10 of the 719 patients $(1.4 \%)$ in the treatment group who received a prophylactic dose of low-molecular-weight heparin during the full period of immobilisation and in 13 of the 716 patients $(1.8 \%)$ in the control group (relative risk, $0.8 ; 95 \% \mathrm{CI}, 0.3$ to 1.7 ; the absolute difference in risk, -0.4 percentage points; $95 \% \mathrm{CI},-1.8$ to 1.0 )with below-knee cast immobilisation as compared with patients with no cast.

Another study was conducted by Giannadakis K in 2000 [8]; the study included 178 patients (118 men, 60 women, mean age 25.8 [16-39] years) who were treated by lower leg plaster for various injuries, including bone and ligamentous. All patient were assessed for thrombosis risk using a checklist which includes age over 40 years, BMI, DM, CHD, History of thrombosis, family history of thrombosis, varicose veins smoking more than 20 cigarettes per day, cancer and degree of soft tissue injuries using Gustilo classification[9]. Then they included only the low-risk group who has any risk from the list mentioned above.

The incidence was $1.1 \%$; consequently, they did not recommend prophylaxis for such patients.

A range of injuries to the lower limb was included in the study, with only 11 patients having an ankle fracture. In addition, the mean duration of immobilisation in a cast was only 14.4 days (5 to 48 ) which did not reflect the usual period of immobilisation in patients with ankle fractures. Patients with risk factors such as obesity and smoking were excluded, and, as such, the reported incidence was not a true reflection of that seen in the general population.

A similar study was done in 2013[16] used a different method of DVT detection, "D Dimer Blood Test," which is not specific to DVT only. In this study,
A total of 95 (77.9\% males) patients with ligament injuries or stable fractures of the foot or ankle were included. The average age was $38 \pm 13.7$ (range 15 $71) .21(22.1 \%)$ patients had positive D-dimer levels. Image Ultrasound Studies showed Venous Thrombosis in only Three out of the Twenty patients $(3.1 \%$ of total patients), and clinical signs and symptoms were present in only one patient.

Additionally, a retrospective study [17] was done on studies between 2005 and 2016, including about 5,200 patients who underwent removable immobilisation; only $16(0.31 \%)$ of them developed VTE. However, this study contains many patients more than ever published though it was limited to symptomatic VTE only and didn't study asymptomatic episodes that went missing.

Routine thromboprophylaxis for all patients with ankle and foot fractures or sprains treated in plaster can be expensive, harmful for the current category of patients where bleeding risk is higher.

According to S. Patil study [7], the incidence of DVT does not warrant routine thromboprophylaxis for everyone, but on the other hand, risk assessment is mandatory to all patients who will undergo lower limb plaster immobilisation for a period of time.

In 2018 Nice [18] ( table 1) issued a new, improved version of VTE assessment and prevention in hospital patients; it is recommended to Assess all patients to identify the risk of venous thromboembolism (VTE) and bleeding, which divided into categories ( medical patients, surgical patients, pregnant women and all women who gave birth or had a miscarriage or termination of pregnancy in the past 6 weeks, people admitted to the critical care unit and all acute psychiatric patients).

Any tick for thrombosis risk should prompt thromboprophylaxis according to NICE guidance. The risk factors identified are not exhaustive. Clinicians may consider additional risks in individual patients and offer thromboprophylaxis as appropriate.

Bleeding risk also should be reviewed, and Any tick should prompt clinical staff to consider if bleeding risk is enough to preclude pharmacological intervention.

In the end, a balance between the person's risk of VTE against their risk of bleeding before deciding whether to offer pharmacological thromboprophylaxis to medical patients.

Immobilisation alone is not a strong enough predisposing factor to affect the incidence of DVT in patients with a short leg plaster. However, the risk of DVT increases significantly in patients with three or more predisposing factors without any risks for bleeding.

For Example, Patients with acute Achilles tendon ruptures may have a high risk of VTE, with reported rates of DVT range from $0.4 \%$ to $34 \%$ without the use of chemoprophylaxis. A Randomized controlled trial during 2014-2016 included a total of 130 patient which divided into two groups $1^{\text {st }}$ EMC group was 
advised to do ankle Rom five times per day from the third week of the injury, The $2^{\text {nd }}$ group in a belowknee cast for eight weeks without ROM, The results showed that there is no difference between the two groups, A total $62(47.7 \%)$ patients were diagnosed with DVT: 33 of those in the ECM group and 28 in the $2^{\text {nd }}$ group.[12]

GENERAL MEASURES should be delivered to all patients having plaster immobilisation: MOBILISATION AND LEG EXERCISES, if tolerated in knee flexion, foot fingers movements, and hip room.

1. HYDRATION should be assured, as Hemoconcentration increases blood viscosity and reduces blood flow, especially in the leg's deep veins in immobile patients.[19]

2. Hormonal contraception. A large epidemiological study suggested around a 2-fold increased risk in women using a combined pill.[20] Interruption of these hormone treatments before planned surgery likely reduces VTE risk, but consideration should be given to the risk of unplanned pregnancy and debilitating menopausal symptoms.

3. Health education about modifiable risk factors such as "smoking, obesity, Hormonal contraceptive pills, and nutrients".

Pharmacological Measure,

The guidelines issued by the American College of Chest Physicians in 2004 advised against routine prophylaxis for patients with isolated injuries of a lower limb [21].

VTE is something can be prevented that's why we need to put extra efforts to avoid it in this study we recommend Thrombosis risk assessment is mandatory checklist protocol to all patient followed by general measure education to all patients and may or may not include chemoprophylaxis in the form of low doses of antiplatelets unless contraindicated for moderate-risk group and injectable or oral anticoagulant for the highrisk group after bleeding risk assessment to each.

\section{Conclusion}

DVT after below-knee cast immobilisation is about 5\%, according to the results of this study, which seems to be low in this category, however, it's very important to put into consideration the risk, morbidity, and mortality, as well as post-thrombotic complication.

\section{References}

[1] L.N.Roberts. Comprehensive VTE Prevention Program Incorporating Mandatory Risk Assessment Reduces the Incidence of HospitalAssociated Thrombosis, 2013.

[2] Using a tool published by a national UK body, professional network or peer-reviewed journal. The most commonly used risk assessment tool for surgical patients is the Department of Health VTE risk assessment tool,2018.

[3] D.R.Anderson, M.Rodger, M.Forgie, C.Kearon,
J.Dreyer, G.Kovacs, M.Mitchell, B.Lewandowski, M.J.Kovacs. Wells' Criteria were derived from a number of studies by Wells, Most recent one which this score is based on is (Evaluation of D-dimer in the diagnosis of suspected deep-vein thrombosis.Wells PS1, Jameson SS, Rankin KS, Desira NL, James P, Muller SD, Reed MR, Rangan A. Pulmonary embolism following ankle fractures treated without an operationan analysis using National Health Service data. Injury.vol. 45(8),pp.1256-1261. ,2014.

[4] P.S.Jorgensen, T.Warming, K.Hansen. Low molecular weight heparin as thromboprophylaxis in outpatients with a plaster cast: a venographic controlled study. Thromb Res.vol.105,pp.477-80,2002.

[5] K.Giannadakis, H.Gehling, H.Sitter. Is a general pharmacologic thromboembolism prophylaxis necessary in ambulatory treatment by plaster cast immobilisation in lower limb injuries? Unfallchirurg.vol. 103,pp.475-8 , 2000.

[6] W.H.Geerts,G.F.Pineo,J.A.Heit.Prevention of venous thromboembolism. Chest.vol.126,pp.338-400,2004.

[7] S.Patil. Incidence of deep-vein thrombosis in patients with fractures of the ankle treated in a plaster cast. J Bone Joint Surg Br.vol.89(10),pp.1340-3,2007.

[8] P.S.Jorgensen. Low molecular weight heparin (Innohep) as thromboprophylaxis in outpatients with a plaster cast: a venografic controlled study. Thromb Res.vol. 105(6),pp.477-80,2002.

[9] A.A.Zee.Low molecular weight heparin for prevention of venous thromboembolism in patients with lower-limb immobilisation. Cochrane Database Syst Rev.vol.8(8),pp. Cd006681,2017.

[10] M.Testroote. Low molecular weight heparin for prevention of venous thromboembolism in patients with lower-leg immobilisation. Cochrane Database Syst Rev.vol.(4),pp.C d006681,2008.

[11] M.Testroote.Low molecular weight heparin for prevention of venous thromboembolism in patients with lower-leg immobilisation. Cochrane Database Syst Rev.vol. (4),pp.Cd006681,2014.

[12]R.A.van Adrichem. Below-knee cast immobilisation and the risk of venous thrombosis: results from a large populationbased case-control study. J Thromb Haemost.vol.12(9),pp.1461-9,2014.

[13]R.A.van Adrichem. Thromboprophylaxis after Knee Arthroscopy and Lower-Leg Casting. New England Journal of Medicine.vol.376(6),pp.515-525, 2016.

[14] K.Giannadakis. [Is a general pharmacologic thromboembolism prophylaxis necessary in 
ambulatory treatment by plaster cast immobilisation in lower limb injuries?]. Der Unfallchirurg.vol.103(6),pp.475-478, 2000.

[15]P.H.Kim, S.S.Leopold. In brief: GustiloAnderson classification. [corrected]. Clinical orthopaedics and related research.vol. 470(11),pp. 3270-3274, 2012.

[16] A.Manafi Rasi. Deep vein thrombosis following below knee immobilisation: the need for chemoprophylaxis. Trauma monthly.vol.17(4),pp.367-369,2013.

[17] R.M.Prince. Symptomatic venous thromboembolism after non-operatively treated foot or ankle injury. J Orthop Res.vol.37(1),pp.190-196,2019.

[18] Nice, Venous thromboembolism in over 16s: reducing the risk of hospital-acquired deep vein thrombosis or pulmonary embolism. 2018.

[19] Alt, E., et al., Blood rheology in deep venous thrombosis - Relation to persistent and transient risk factors. Thrombosis research, 2002. 107: p. 101-7.

[20] Vessey, M., et al., Oral contraceptives and venous thromboembolism: findings in a large prospective study. British medical journal (Clinical research ed.), 1986. 292(6519): p. 526-526.

[21] Geerts, W.H., et al., Prevention of venous thromboembolism: the Seventh ACCP Conference on Antithrombotic and Thrombolytic Therapy. Chest, 2004. 126(3 Suppl): p. 338S-400S. 